\title{
LE REGIME ALIMENTAIRE DE DINOCRAS MEGACEPHALA (KLAPALEK) [PLECOPTERA, PERLIDAE]
}

\author{
par J. MOUTHON ${ }^{2}$ et J. VERNEAUX ${ }^{1}$
}

Une population de Dinocras megacephala a été étudiée dans le cours supérieur de la Loue. Le cycle larvaire est de trois années. La larve se développe préférentiellement au sein des bryophytes et migre en direction des berges peu avant l'éclosion.

Le régime alimentaire à tendance végétale et détritivore devient rapidement carnivore lorsque la larve dépasse $3 \mathrm{~mm}$. L'ingestion de proies de nature différente est en relation directe avec la succession des abondances spécifiques maximales d'individus atteignant la "taille moyenne de prédation".

Le schéma des relations trophiques de ce prédateur met l'accent sur l'importance du facteur nutritif dans la compréhension des biocénoses.

Une nouvelle espèce de Sporozoaire parasite du tube digestif de $D$. megacephala: Ancyrophora cornuta (Gregarinida) a été découverte.

\section{The diet of Dinocras megacephala (Klapalek) (Plecoptera, Perlidae).}

The plecopteran Dinocras megacephala has a life cycle that lasts three years in the upper part of the river Loue. Larvae generally live in mosses. Just before hatching, they migrate to the banks.

The young larvae feed on plants and detritus. The diet is carnivorous when larvae becomes longer than $3 \mathrm{~mm}$.

The rate at which the predator consumes prey is directly conrelated with a required " mean length of predation".

The results of this study suggest that diets is an important item for the understanding of biocenoses.

A new Sporozoaire: Ancyrophora cornuta (Gregarinida) was discovered in the alimentary duct of Dinocras megacephala.

D'après Despax (1951), Bertrand (1954), Illies (1955), Aubert (1959), on sait que les larves de Plécoptères de la famille des Perlidae ont un régime général carnivore. Toutefois, à notre connaissance, aucune étude détaillée n'a été réalisée sur le régime alimentaire des Perlidae européens. Nous proposons l'examen du cas de D. megacephala (Klapalek 1907), espèce considérée comme alpine (Aubert 1954), assez largement distribuée en France - zones 4, 8, 13 de la Limnofauna Europaea (Illies 1967) et relevés personnels -

1. Lab. Hydroécol. Division Qualité des Eaux du C.T.G.R.E.F. - Centre Hydrobiol. Institut des Sciences Naturelles, place Leclerc, 25042 Besançon.

2. D'après un travail de D.E.A. soutenu en octobre 1975. 
Les prélèvements de faune ont été effectués dans la Loue supérieure au lieu dit "les jardins de Mouthier " situés en contrebas du village de Mouthier-Hautepierre. Pour la description de cette station on se reportera aux travaux effectués antérieurement par l'un de nous (Verneaux 1973-74).

\section{1. - MATERIEL ET METHODES}

Les prélèvements mensuels ont porté sur une année, exception faite des mois d'octobre et novembre, pendant lesquels la Loue demeura en crue ; ils ont été effectués à l'aide d'un filet échantillonneur classique de $1 / 10 \mathrm{~m}^{2}$ de surface de base présentant une maille de $250 \mu$. La portion de support ainsi délimitée était prospectée sur $5 \mathrm{~cm}$ d'épaisseur et les invertébrés récoltés fixés au formol (solution aqueuse à $7 \%$ ).

Tous les individus de l'espèce considérée ont été mesurés puis disséqués, le contenu du tube digestif étant dispersé dans une goutte d'eau glycérinée puis observé entre lame et lamelle au microscope.

L'identification des proies a été effectuée par comparaison à des montages préalables des espèces peuplant la station; dans le cas des Oligochètes, lorsqu'il ne reste que des soies éparses du même type, nous avons considéré qu'il n'y en avait qu'un par tube digestif (Tachet 1965).

\section{2. - CYCLE BIOLOGIQUE}

Le cycle biologique de Dinocras megacephala, de trois années (Aubert 1959), fut contrôlé par la mesure de la longueur médiane du mésonotum (Tableau I).

A l'altitude considérée, la période d'éclosion des adultes est longue : de début avril à fin septembre, en 1974. En revanche au cours de l'année 1975, dans des conditions climatiques difficiles, les émergences n'ont commencé que début mai. Pour l'ensemble du bassin, les dates extrêmes de période de vol s'étalent du 10 avril au 7 octobre (Verneaux 1973). L'éclosion des adultes suit de près l'étalement des feuilles des arbres bordant la rivière, même lorsque l'éclatement des bourgeons est retardé, comme ce fut le cas en 1975. Ce synchronisme n'est pas dépourvu d'intérêt, les feuilles constituant l'abri principal des imagos.

La période d'éclosion des larvules s'étale du début de l'automne à la fin du printemps. La croissance des larves, rapide de juillet à septembre, est lente le reste de l'année; après un an, la larve atteint une taille d'environ $10 \mathrm{~mm}$. 


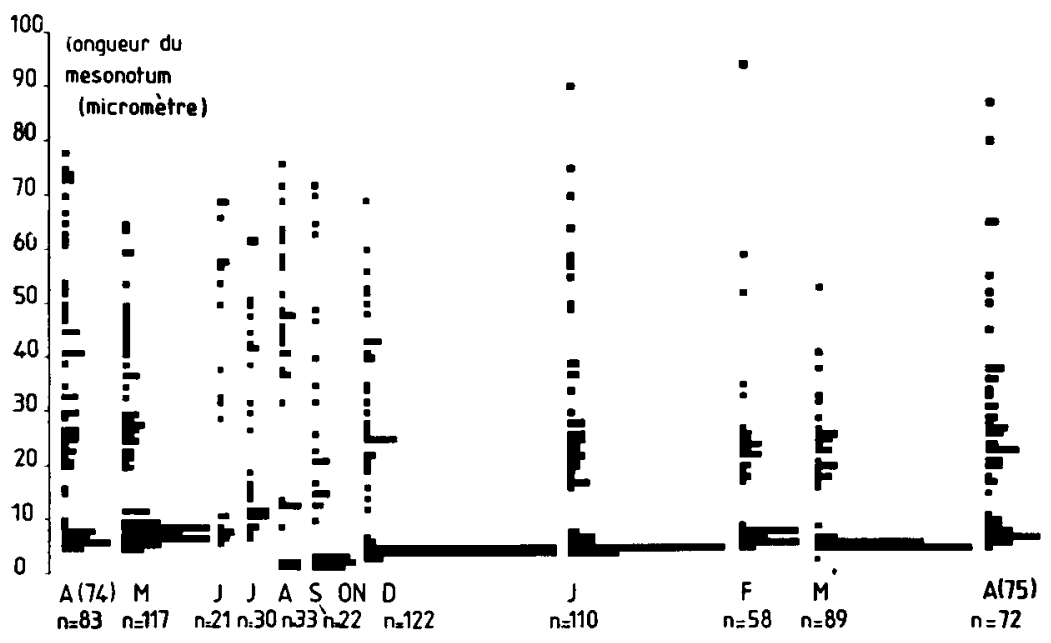

1 individu

Tableau I. - Cycle biologique de Dinocras megacephala Klapalek.

\section{3. - HABITAT}

Le développement des larvules s'effectue exclusivement dans les bryophytes: 130 individus de taille inférieure à $3 \mathrm{~mm}$ pour $100 \mathrm{~cm}^{3}$ de Cinclidotus fontinaloïdes et aquaticus (espèces généralement en courant moyen ou fort). Dès la fin mai, on observe une importante concentration des larves âgées (classes de taille 4 et 5 ) sous les gros galets et les pierres situés en bordure de la rivière dans des vitesses de courants faibles ou presque nulles. En soulevant l'un d'eux, il est très fréquent d'observer, agglomérés à la faveur d'un évidement de la roche, 8 à 12 larves prêtes à éclore. Du début de cette migration jusqu'à l'éclosion de l'adulte, la larve ne prend plus aucune nourriture.

\section{4. - ALIMENTATION DE LA LARVE}

\section{LA LARVUle $: \leqslant 3 \mathrm{~mm}$}

Les larvules se nourrissent principalement de Diatomées et de particules inertes comme l'attestent les pourcentages importants de granules sableux et d'éléments organiques détritiques rencontrés dans leurs tubes digestifs. Elles ont donc un régime alimentaire composite à tendance végétale et détritivore. Toutefois, si la participation de Protozoaires à la nutrition de $D$. megacephala n'a pu être établie avec certitude, il n'en est pas de même pour les Tardigrades et les Roti- 
fères, identifiés grâce à leur mastax chitinisé. Bien que saisonnière, la contribution de ces invertébrés au régime alimentaire des larvules n'est pas négligeable.

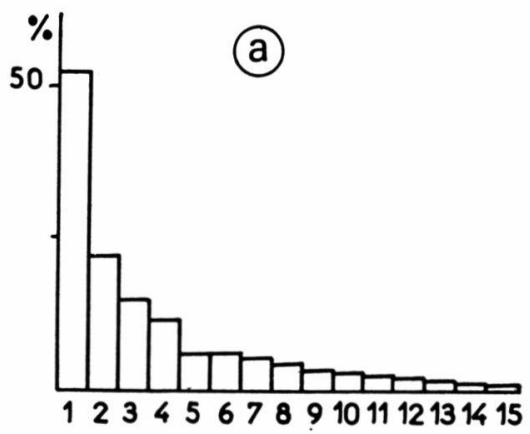

1 Orthocladiinae
2 Tanytarsini
3 Baetis
4 Autres Chironomidae
5 Protonemura
6 0ligochètes
7 Trichoptères
8 Heptageniidae

\author{
9 Simuliidae \\ 10 Ephemerella \\ 11 Hydracariens \\ 12 Coléoptères \\ 13 Leuctra \\ 14 Leptophlebiidae \\ 15 Crustacês
}

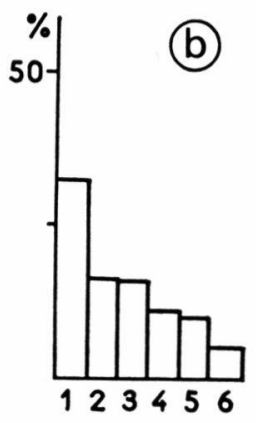
1 Diatomées
2 Audouinella
3 Bryophytes
4 Tribonena, Vaucheria
5 Microspora
6 Chlorogloea

Fig. 1. - a : Proportions relatives exprimées en pourcentages de chacun des groupes d'inventébrés consommés par rapport au nombre total de prédateurs. $\mathrm{b}$ : Propontions relatives, exprimées en pourcentages, des principaux végétaux consommés, par rapport au nombre de tubes digestifs analysés.

\section{LA LARVE: $\geqslant 3 \mathrm{~mm}$}

Chironomides, Ephéméroptères et Oligochètes servent de base à l'alimentation des larves de $D$. megacephala. On rencontre encore, mais avec des pourcentages plus faibles, des Plécoptères, des Trichoptères sans fourreau, des Hydracariens, peu de Coléoptères (fig. $1 \mathrm{a}$ et $1 \mathrm{~b})$.

Le prélèvement annuel global effectué par les 685 individus récoltés ne représente que $0,65 \%$ de la population recensée sur la station; la faiblesse relative de ce pourcentage est due à l'importance numérique des populations de Chironomides dans l'effectif stationnel. En outre, on note fréquemment dans le bol alimentaire des larves, la présence de végétaux (algues et mousses) très irrégulièrement consommés. Les éléments siliceux (frustules de Diatomées), cellulosiques (parois cellulaires) et chitinisés sont rejetés.

\section{INTENSITÉ DE LA PRÉDATION}

L'intensité de la prédation croît avec la classe de taille et les lar- 
vules de la première classe présentent le plus fort pourcentage d'estomacs vides ( $64 \%$, fig. 2 c) ; importante au printemps et en été (fig 2 a), en accord avec la croissance rapide des larves durant cette période, l'intensité de prédation est minimum en hiver. Le nombre moyen de proies par prédateur est de 3,8 (fig. 2 b).

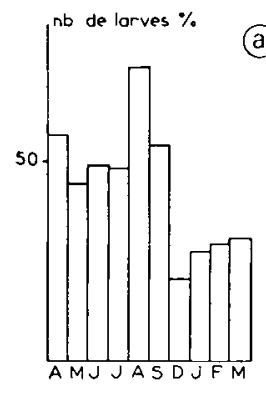

(a)

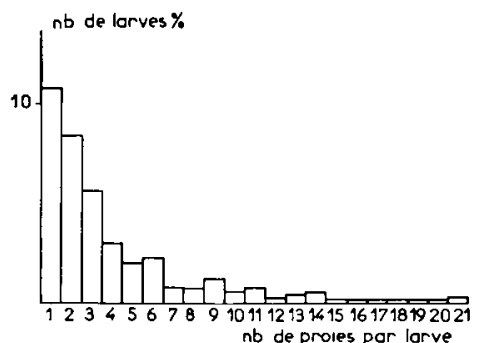

(c)

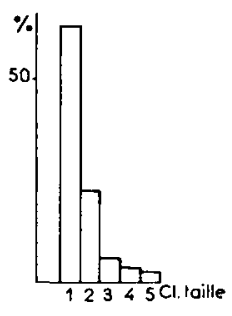

FIG. 2. - a: Histogramme de distribution du nombre de Dinocras megacephala Klapalek ayant au moins une proie par tube digestif. b: Histogramme de distribution du nombre de proies ingérées par les larves de Dinocras megacephala Klapalek. c: Proportions, exprimées en pourcentages, du nombre de tubes digestifs vides par classe de taille.

\section{5. - VARIATIONS SAISONNIERES, RELATIONS PREDATEUR-PROIES}

Les variations saisonnières du régime alimentaire de $D$. megacephala se traduisent par une modification de la nature des proies ingérées (fig. $3 \mathrm{a}, \mathrm{b}, \mathrm{c}$ ).

Il existe une corrélation positive hautement significative entre la taille du prédateur et celle des proies qu'il s'agisse de Plécoptères, de Trichoptères ou d'Ephéméroptères (fig. 4 a). La succession des proies de ces différents ordres correspond, pour chaque espèce ou genre considéré, à la présence optimale d'individus d'une taille bien définie que l'on pourrait appeler « taille moyenne de prédation " évaluée, par exemple, pour Baetis alpinus et $B$. rhodani à $2,7 \mathrm{~mm}$, pour Ephemerella ignita à $2,8 \mathrm{~mm}$. Les pourcentages d'individus des différentes tailles correspondant à la fourchette de prédation sont indiqués dans les tableaux ci-dessous:

\begin{tabular}{lccc}
\hline \multirow{2}{*}{ Mois } & Baetis alpinus & tailles & (classes) $\mathrm{mm}$ \\
& $\leqslant 1$ & $2-3-4$ & $\geqslant 5$ \\
\hline mars & $71,0 \%$ & $23,0 \%$ & $5,6 \%$ \\
avril & $19,4 \%$ & $77,0 \%$ & $3,0 \%$ \\
mai & $19,4 \%$ & $57,3 \%$ & $22,7 \%$ \\
juin & $0,0 \%$ & $32,0 \%$ & $68,0 \lessgtr$ \\
\hline
\end{tabular}



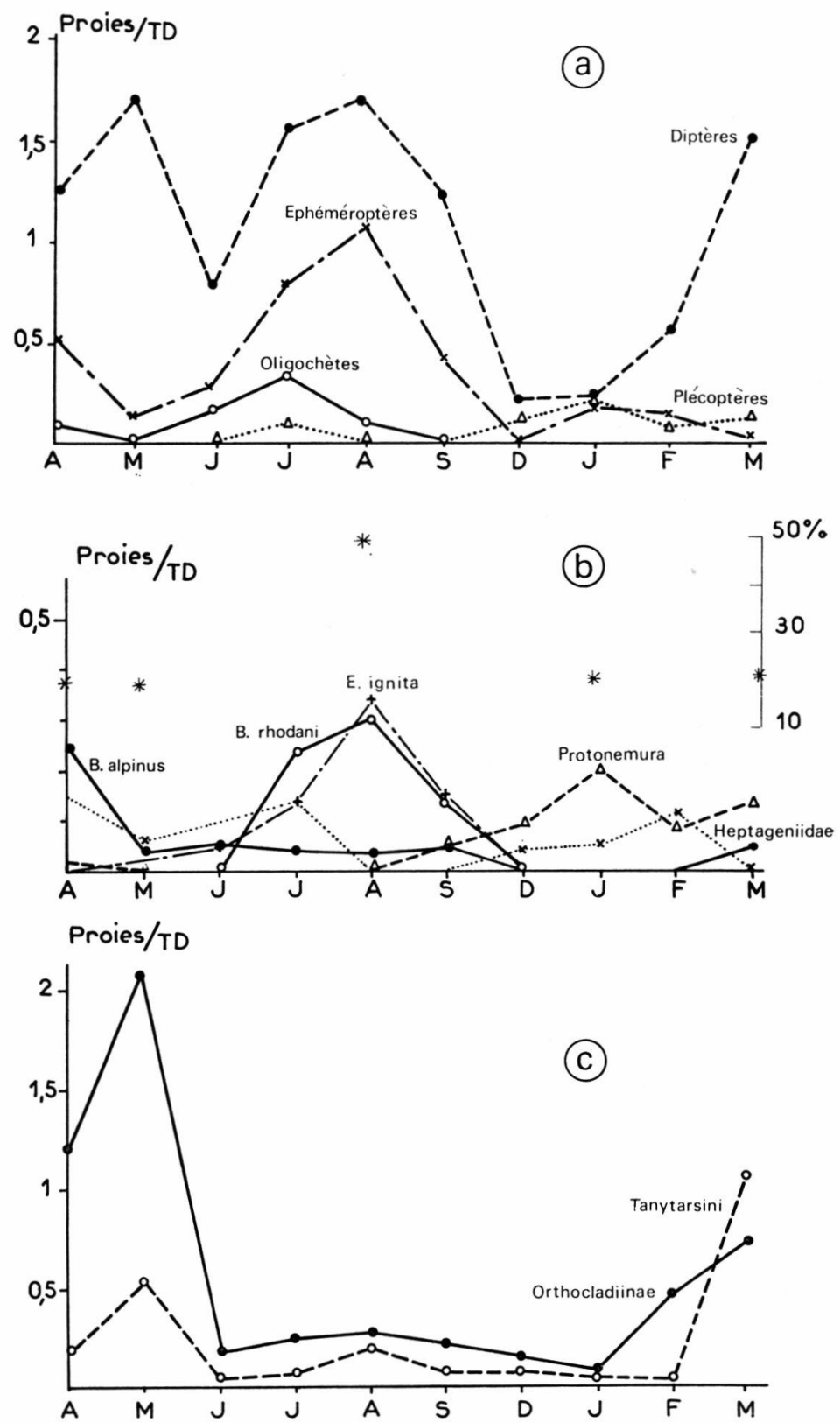

Fig. 3. - a b c: Variations saisonnières du contenu stomacal exprimées par le rapport du nombre de proies dénombrées au nombre d'estomacs analysés. *: représente les maximums de la variation des pourcentages de tubes digestifs contenant des mousses. 


\begin{tabular}{lccc}
\hline \multirow{2}{*}{ Mois } & Ephemerella ignita & tailles & (classes) $\mathrm{mm}$ \\
& $\leqslant 1$ & $2-3-4$ & $\geqslant 5$ \\
\hline mai & $63 \%$ & $27 \%$ & $0 \%$ \\
août & $4 \%$ & $60 \%$ & $36 \%$ \\
septembre & $0 \%$ & $36 \%$ & $54 \%$ \\
\hline
\end{tabular}

Les pourcentages maximums pour ces deux espèces, prises comme exemple, correspondent bien à une intensité maximale de la prédation
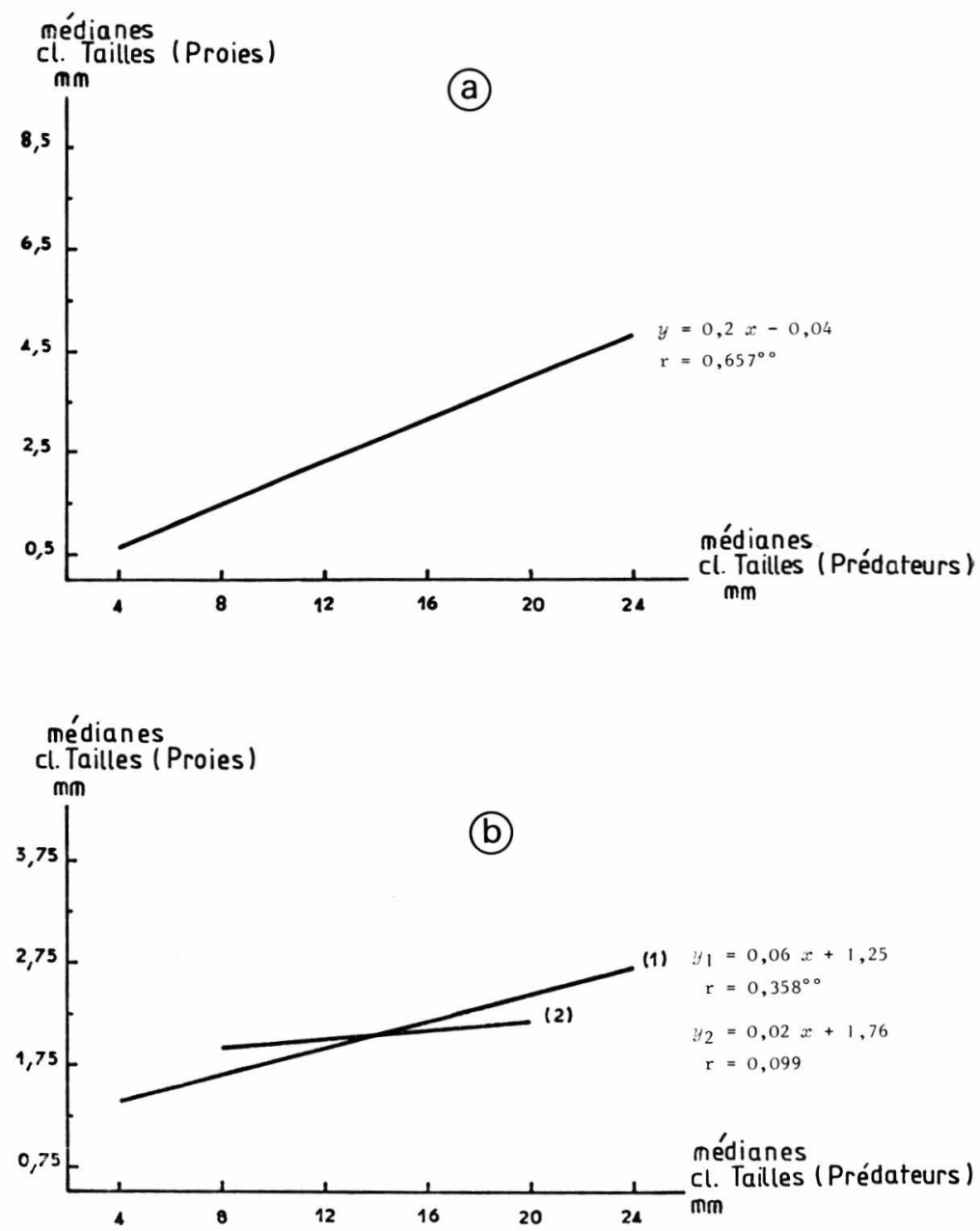

Fig. 4. - a : Relation entre la taille des proies (Ephéméroptères) et celle du prédateur. b: Relation entre la taille des proies (Chironomides) et celle du prédateur.

(1) : en considérant toutes les classes de taille.

(2) : en excluant les classes extrêmes de taille. 
exercée par Dinocras respectivement sur B. alpinus en avril, sur E. ignita en août.

On peut donc conclure avec Tachet (1965) que «l'abondance de larves d'Ephéméroptères de taille convenable semble être le facteur essentiel de leur abondance dans les tubes digestifs». En ce qui concerne les Chironomides, la corrélation n'est plus significative lorsque l'on exclut les deux classes extrêmes de taille du prédateur (individus de 2 à $6 \mathrm{~mm}$ et de 22 à $26 \mathrm{~mm}$ ). On peut donc en déduire que pour les Dinocras mesurant de 6 à $22 \mathrm{~mm}$, il n'y a pas de relation directe entre la taille des proies et celle du prédateur (fig. $4 \mathrm{~b}$ ). D'une façon générale, plus les proies consommées sont de forte taille plus la fréquence de consommation est faible.

La partie vétégale de la nourriture de $D$. megacephala ne semble pas présenter de variations marquées. La consommation des différents végétaux, algues et mousses, est toutefois étroitement liée aux cycles biologiques de ces différents groupes floristiques. Le fait que seul le tiers supérieur des feuilles de mousse soit le plus souvent trouvé dans les tubes digestifs laisse à penser que l'apport végétal dans le régime alimentaire est accidentel et résulte du comportement prédateur de l'espèce : ainsi tous les pics de la courbe de consommation des mousses correspondent aux pics de consommation maximale des espèces numériquement bien représentées dans ce biotope: $B$. alpinus, E. ignita, Protonemura sp. (fig. 3 b). L'apport végétal constitue donc ce que l'on peut appeler une "nourriture obligatoire "due à la voracité de l'espèce, celle-ci ne pouvant être qualifiée de carnivore stricte.

\section{SÉLECTION DES PROIES}

En appliquant un coefficient de sélection "forage ratio » (HessSwartz 1940) ou d' "avaibility factor" (Allen 1941) exprimé par le rapport :

$$
\mathrm{FR}=\frac{\% \text { de proie } x \text { dans le tube digestif du prédateur }}{\% \text { de cette proie } x \text { dans le milieu }}
$$

on estime que si $\mathrm{FR}<1$ : le prédateur dédaigne la proie $x$

$\mathrm{FR}=1:$ le prédateur absorbe au hasard la proie $x$

FR $>1$ : le prédateur choisit la proie $x$.

Cette "sélection apparente" ne permet toutefois pas de faire la part entre le choix du prédateur et la disponibilité de la proie (Dumont et Verneaux 1976). 


\section{Proies FR}

$\begin{array}{llll}\text { Baetis } & \mathbf{5 , 3} & \text { Oligochètes } & 2,5 \\ \text { Ephemerella } & 1 & \text { Hydracariens } & 1,4 \\ \text { Heptageniidae } & 5 & \text { Coléoptères } & 0,55 \\ \text { Protonemura } & 3,3 & \text { Trichoptères } & 8,4 \quad \text { (sans fourreau) } \\ \text { Leuctra } & 0,75 & \text { Simuliidae } & 3,8 \\ & & \text { Chironomides } & 2\end{array}$

Leuctra et Coléoptères semblent être dédaignés, Ephemerella, Hydracariens, Protonemura, Simuliidae, mais surtout Chironomides et Oligochètes sont absorbés plutôt au hasard; en revanche le choix de Dinocras, correspondant à un effort de capture, s'exercerait particulièrement sur les Heptageniidae, Baetis et Trichoptères sans fourreau (Hydropsyche et Rhyacophila).
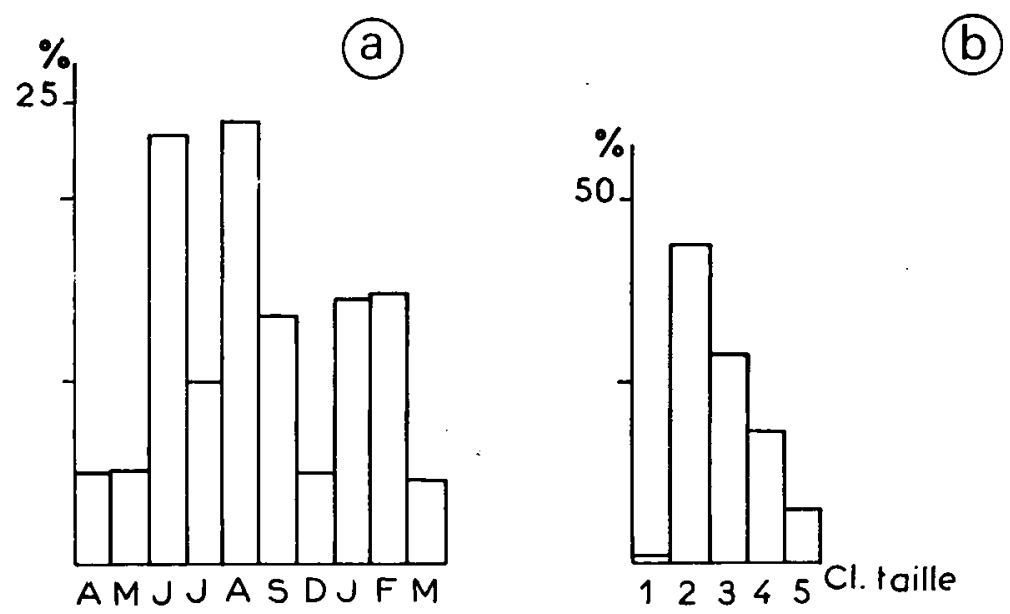

Fig. 5. - a : Variations saisonnières du pourcentage de Dinocras megacephala

Klapalek parasités. b : Variation du parasitisme dans les différentes classes de taille de Dinocras megacephala Klapalek.

\section{PARASITISME}

L'analyse des contenus stomacaux de $D$. megacephala a révélé la présence, chez $12 \%$ des individus examinés, d'une nouvelle espèce de Grégarine (ordre des Sporozoaires) : Ancyrophora cornuta (Baudoin et Mouthon 1976).

Le parasitisme des larves est loin d'être uniforme (fig. $5 \mathrm{a}, 5 \mathrm{~b}$ ) ; en effet, les larvules de la classe 1 sont dépourvues de parasites, alors que celles de la classe 2 , qui deviennent franchement carnivores, sont fortement parasitées. 
Cette observation confirme les conclusions des travaux de Baudoin (1967-1971), Baudoin et Maillard (1972), mettant en évidence l'étroite dépendance qui existe entre le mode de fixation des Grégarines et le régime de leurs hôtes. En l'occurrence le genre Ancyrophora paraît strictement inféodé à des hôtes carnivores.

\section{6. - DISCUSSIONS ET CONCLUSIONS}

L'examen de la participation des différents groupes faunistiques présents sur la station à l'alimentation des larves de $D$. megacephala conduit à effectuer certaines remarques :

- les Leuctra, Plécoptères pétricoles, son peu consommés,

- les Caenidae, aux mœurs fouisseuses, échappent totalement à la prédation exercée par Dinocras,

- les Heptageniidae, seulement rencontrés dans les tubes digestifs des plus grosses larves de Dinocras, ne dépassent pas $8 \mathrm{~mm}$; l'aplatissement dorso-ventral des représentants de cette famille semble constituer une protection efficace contre la prédation,

- aucun Trichoptère à fourreau ne paraît consommé,

- les Diptères chironomides constituent l'alimentation principale de Dinocras; en effet la taille et la morphologie de ces Diptères permettent aux larves prédatrices de taille supérieure à $3 \mathrm{~mm}$ de trouver une nourriture abondante, disponible, particulièrement dans les Bryophytes,

- la non-consommation des Coléoptères adultes est sans doute en relation avec la présence d'une cuticule protectrice (Maitland 1963).

Il semble donc que Dinocras se nourrisse préférentiellement au sein des Bryophytes dans lesquels les organismes de petite taille (Chironomides, Protonemura, Ephemerella, Baetis, Tardigrades et Rotifères) effectuent leur développement. Les larves nymphes, se rassemblant sur les pierres de la zone rivulaire en courant faible, ne s'alimentent pas avant l'éclosion. D'une manière générale, il est intéressant de constater que seul l'aspect quantitatif varie entre les régimes alimentaires d'espèces européennes telles que Perla carlukiana Klapálek (Mackereth 1957), Dinocras (Perla) cephalotes Curtis (Chisholm 1962), et d'espèces américaines telles que Acroneuria californica Banks (Sheldon 1969), A. pacifica Banks (Richardson et Gaufin 1971). On retrouve en effet les mêmes groupes de proies pour ces cinq espèces et la présence de nourriture végétale est quasi constante.

Le régime alimentaire des larvules est rarement précisé par les auteurs; Chisholm (1962) cependant, signale la présence de frustules de Diatomées dans les tubes digestifs des premiers stades larvaires de D. cephalotes. 


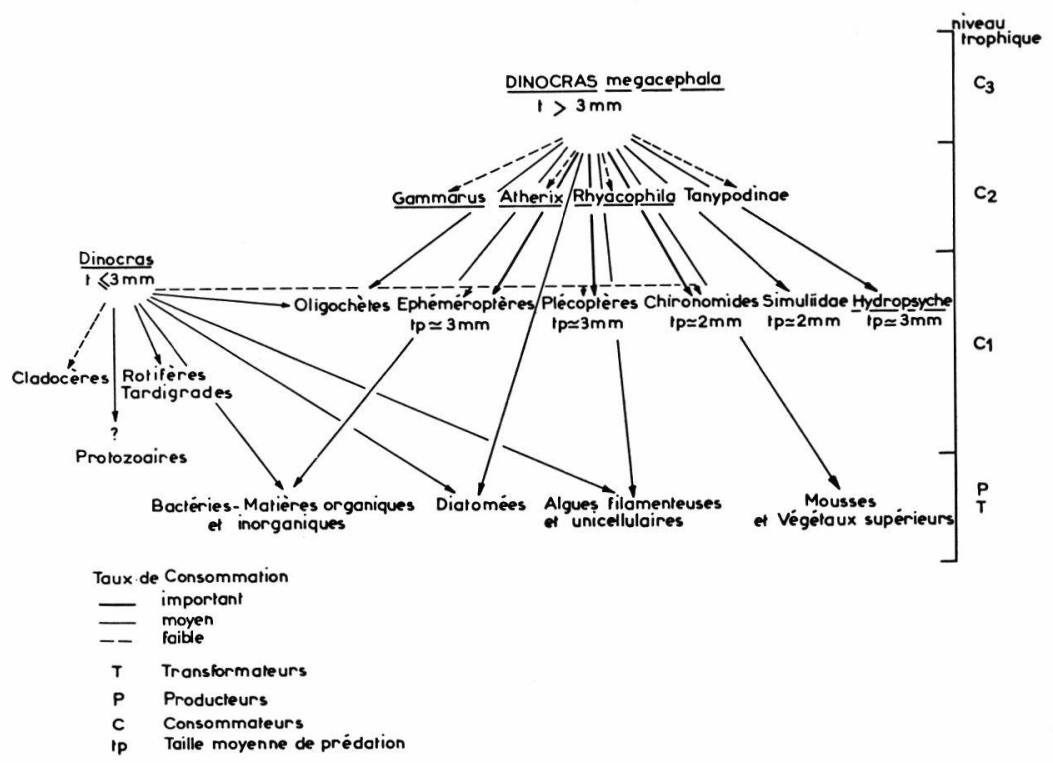

TABLEAU II. - Relations trophiques de Dinocras megacephala Klapalek.

La larve de $D$. megacephala occupe donc plusieurs niveaux trophiques, sa niche écologique évoluant au cours de son développement larvaire. Ces niveaux traditionnellement dénommés $\mathrm{C} 1, \mathrm{C} 2, \mathrm{C} 3$ ne tiennent compte ni des Bactéries, ni des Protozoaires, ni de microinvertébrés tels que Rotifères, Tardigrades, etc. (Tableau II). La composition du régime alimentaire de cette espèce, qui peut être qualifiée de carnivore à tendance microphage est, aux différentes saisons et aux différentes étapes du développement larvaire, conditionnée par l'accessibilité des éléments disponibles de la biocénose du site.

\section{TRAVAUX CITES}

AlLEN (K. R.). 1941. - Studies on biology of the early stages of the Salmon (Salmo salar). 2. Feeding habits. J. anim. Ecol., 10 : 47-75.

Aubert (J.). 1959. - Plecoptera. Insecta Helvetica Fauna. Lausanne, 139 p.

Bertrand (H.). 1954. - Les insectes aquatiques d'Europe. Tome 2. Lechevalier, Ed. Paris, $547 \mathrm{p}$.

Baudouin (J.). 1967. - Contribution à l'étude morphologique, biologique et écologique des Grégarines d'insectes à larves aquatiques. Ann. Station Biol. Besse., $2: 13-160$.

Baudorn (J.). 1971. - Etude comparée de quelques Grégarines Acanthosporinae. J. Protozool., 18 (4) : 654-660.

Baudoin (J.) et MaillaRd (Y.P.). 1972. - Recherches sur les Grégarines des Coléoptères hydrophiles. Protistologica. VIII (1) : 53-63. 
Baudorn (J.) et Mouthon (J.). 1976. - Nouvel exemple de corrélation entre la présence d'une Grégarine du genre Ancyrophora Leger (1892), et le régime alimentaire de I'hôte. C. R. Acad. Sc., t. 282, série D, p. 1285-1287.

CHISholm (P.J.). 1962. - The anatomy in relation to feeding habits of Perla cephalotes Curtis. (Plecoptera, Perlidae) and other Plecoptera. Trans. Soc. Brit. Ent., 15 (5) : 55-101.

Despax (R.), 1951. - Plécoptères. In Faune de France. 55 : Lechevalier, Ed., Paris, $280 \mathrm{p}$.

Dumont (B.) et Verneaux (J.). 1976. - Edifice trophique partiel du cours supérieur d'un ruisseau forestier. Annls Limnol., 12 (3) : 239-252.

Hess (A. D.) et Swartz (A.). 1940. - The forage ratio and its use in determining the food graide of stream. Trans. 5th N. Amer. Wildlife Conf., 162-164.

Illies (J.). 1955. - Steinfliegen oder Plecoptera. Die Tierwelt Deutschlands. 43, Gustav. Fisher Verlag, Jena, 150 p.

Illies (J.). 1967. - Plecoptera. In Limnofauna Europaea. Gustav Fisher Verlag, Stuttgart, $474 \mathrm{p}$.

JoNEs (J. R. E.). 1950. - A further ecological study of the river Rheidol : the food of the common insects of the Mainstream. J. anim. Ecol., 19:159-174.

Mackereth (J.). 1957. - Notes on the Plecoptera from a stony stream. J. anim. Ecol., $26: 343-351$.

MaItland (P. S.). 1967. - The ecology of four species of Elminthidae in a Scottish river. Arch. Hydrobiol. 63 (1): 104-122.

Mouthon (J.). 1975. - Dinocras megacephala (Klapalek, 1907) cycle biologique, régime alimentaire et ses variations. D.E.A. Fac. Sci. Lyon, 28 p.

Muller-Liebenau (I.). 1969. - Revision der europaïschen Arten der Gattung Baetis Leach, 1815. (Insecta Ephemeroptera). Gew. und $A b w ., 48-49: 214$ p.

Richardson (J. W.) et Gaufin (A. R.). 1971. - Food habits of some western Stonefly nymphs. Trans. Amer. Ent. Soc., $97: 91-121$.

SHELdon (A. L.). 1969. - Size relationships of Acroneuria californica (Perlidae, Plecoptera) and its prey. Hydrobiologia., 34 (1) : 85-94.

TACHET (H.). 1965. - Recherches sur l'alimentation des larves de Polycentropus (Trichoptères) dans leur milieu naturel. Ann. Soc. Ent. Fr. N.S., 1 (3) : 627633.

Verneaux (J.). 1973. - Recherches écologiques sur le réseau hydrographique du Doubs. Essai de biotypologie. Ann. Sci. Univ. Besançon, 9:260 p.

Verneaux (J.) et Rezzouk (M.). 1974. - Les structures d'un grand cours d'eau à Salmonidés : la Loue (Massif du Jura) - Essai typologique et problèmes des relations entre espèces et milieu. Annls. Limnol., 10 (2) : 131-162. 